International Journal of Pure and Applied Mathematics

Volume 111 No. 1 2016, 43-53

ISSN: 1311-8080 (printed version); ISSN: 1314-3395 (on-line version)

url: http://www.ijpam.eu

doi: 10.12732/ijpam.v111i1.5

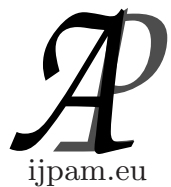

\title{
TERNARY PARTIAL SEMIRINGS
}

\author{
K. Siva Prasad ${ }^{1}$, Y. Prabhakara Rao ${ }^{2}$ \\ ${ }^{1}$ Department of Mathematics \\ Acharya Nagarjuna University \\ Nagarjuna Nagar, 522510, Guntur (D.T), Andhra Pradesh, INDIA \\ ${ }^{2}$ Department of Science \& Humanities \\ Vasireddy Venkatadri Institute of Technology \\ Nambur, Guntur, 522510, Andhra Pradesh, INDIA
}

\begin{abstract}
In this paper, the notion of ternary partial semiring is introduced and several examples of ternary partial semirings are given. Also, congruence relations and quotients of ternary partial semirings are studied.
\end{abstract}

AMS Subject Classification: $16 \mathrm{Y} 60$

Key Words: ternary partial semiring, ternary partial sub-semiring, homomorphism, ternary partial semiring congruence relation, quotient

\section{Introduction}

Partially defined infinitary operations occur in the contexts ranging from integration theory to programming language semantics. Many authors studied various types of semirings. $\Sigma$-structures were studied by Higgs in 1980. Arbib and Manes [3], [11] introduced partial monoids and sum ordered partial monoids in 1980. Partial semirings were studied by Streenstrup [12] in 1985. A partial semiring is a quadruple $(R, \Sigma, \cdot, 1)$, where $(R, \Sigma)$ is a partial monoid, $(R, \cdot, 1)$ is an ordinary monoid with multiplicative binary operation '. ' and unit 1 , and the additive and multiplicative structures obey the both distributive laws.

Received: June 12, 2016

Revised:

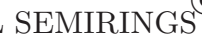

Published:

December 2, 2016

2016 Academic Publications, Ltd.

url: www.acadpubl.eu 
The begining of ternary algebra was started before 1932. In 1932, D. H. Lehmer [8] investigated certain ternary algebraic systems called triplexes which turned out to be ternary groups. In 1971, Lister [9] introduced the notion of ternary rings and studied some of their properties. In 2003, Dutta and Kar [5] introduced the notion of ternary semiring which is a generalization of ternary ring.

In this paper, the notion of ternary partial semiring is introduced and the concept of quotient structure on ternary partial semiring is studied. A necessary and sufficient condition for $S / \theta$ to be ternary partial semiring is obtained, where $\mathrm{S}$ is a ternary partial semiring and $\theta$ is a congruence relation on $\mathrm{S}$.

\section{Preliminaries}

Let $M$ be a nonempty set and let $I$ be a set. An $I$-indexed family in $M$ is a function $x: I \rightarrow M$, denoted by $\left(x_{i}: i \in I\right)$ where $x_{i}=i x$ for each $i$ in $I$. Two families $\left(x_{i}: i \in I\right)$ and $\left(y_{j}: j \in J\right)$ are isomorphic if there is a bijection $\sigma: I \rightarrow J$ with $y_{i \sigma}=x_{i}$ for each $i$ in $I$. A subfamily of $\left(x_{i}: i \in I\right)$ is a family $\left(x_{k}: k \in K\right)$ such that $K \subseteq I$. The empty family is indexed by $\emptyset$. A family $\left(x_{i}: i \in I\right)$ in $\mathrm{M}$ infinite (finite) if the cardinality of the index set $\mathrm{I}$ is infinite (finite).

Now we consider an infinitary operation $\Sigma$ which takes (finite or infinite) families in $M$ to elements of $M$, but which may not be defined for all families in $M$. As $\Sigma\left(x_{i}: i \in I\right)$ need not be defined for an arbitrary family $\left(x_{i}: i \in I\right)$ in $M, \Sigma$ is partially defined. We say that a family $\left(x_{i}: i \in I\right)$ in $M$ is summable if $\Sigma\left(x_{i}: i \in I\right)$ is defined and is in $M$. We use the notations $\Sigma\left(x_{i}: i \in I\right), \Sigma_{i \in I} x_{i}$ and $\Sigma_{i} x_{i}$ inter-changeably.

Definition 2.1. A partial monoid is a pair $(M, \Sigma)$ where $M$ is a nonempty set and $\Sigma$ is a partial addition defined on some, but not necessarily all families $\left(x_{i}: i \in I\right)$ in $M$ subject to the following axioms:

(1) Unary Sum Axiom. If $\left(x_{i}: i \in I\right)$ is a one element family in $M$ and $I=\{j\}$, then $\Sigma\left(x_{i}: i \in I\right)$ is defined and equals $x_{j}$.

(2) Partition-Associativity Axiom. If $\left(x_{i}: i \in I\right)$ is a family in $M$ and $\left(I_{j}: j \in J\right)$ is a partition of $I$, then $\left(x_{i}: i \in I\right)$ is summable if and only if $\left(x_{i}: i \in I_{j}\right)$ is summable for every $j$ in $J$ and $\left(\Sigma\left(x_{i}: i \in I_{j}\right): j \in J\right)$ is summable and then $\Sigma\left(x_{i}: i \in I\right)=\Sigma\left(\Sigma\left(x_{i}: i \in I_{j}\right): j \in J\right)$.

The following are consequences of the partition associativity axiom:

(i) $\Sigma$ is an associative and a commutative operation. 
(ii) Any two isomorphic families have the same sum.

(iii) Every sub-family of a summable family is itself summable.

(iv) There do not exist any nontrivial additive inverses.

Observation 2.2. In a partial monoid $(M, \Sigma)$, the empty family is summable. Its sum, denoted by 0 , is such that the sum of an arbitrary number of 0 's is itself equal to 0 . Furthermore ' 0 ' acts as an additive zero in $M$.

Definition 2.3. The support of a family $\left(x_{i}: i \in I\right)$ in $M$ is defined to be the subfamily $\left(x_{i}: i \in J\right)$ where $J=\left\{i \in I \mid x_{i} \neq 0\right\}$.

Observation 2.4. In a partial monoid $(M, \Sigma)$, if $\Sigma\left(x_{i}: i \in I\right)$ is defined and equals 0 , then $x_{i}=0$ for all $i$ in $I$.

Example 2.5. Let $D, E$ be sets and let the set of partial functions from $D$ to $E$ be denoted by $p f n(D, E)$. Then $(p f n(D, E), \Sigma)$ is a partial monoid if $\Sigma$ is defined such that a family $\left(x_{i}: i \in I\right)$ is summable if and only if for $i, j$ in $I$, and $j \neq i, \operatorname{dom}\left(x_{i}\right) \cap \operatorname{dom}\left(x_{j}\right)=\emptyset$, where $\operatorname{dom}(f)$ is the domain of definition of the partial function $f$. If $\left(x_{i}: i \in I\right)$ is summable then for any $d$ in $D$,

$$
d\left(\Sigma_{i} x_{i}\right)=\left\{\begin{array}{l}
d x_{i}, \text { if } d \in \operatorname{dom}\left(x_{i}\right) \text { for some (necessarily unique) } i \in I \\
\text { undefined, otherwise. }
\end{array}\right.
$$

Example 2.6. Let $D, E$ be sets. A multifunction $x: D \rightarrow E$ maps each element in $D$ to an arbitrary subset of $E$. Such multifunctions correspond bijectively to relations $R \subseteq D \times E$, where $(d, e) \in R$ if and only if $e \in d x$. The set of multifunctions from $D$ to $E$, denoted by $M f n(D, E)$, together with $\Sigma$ defined such that for $d$ in $D, d\left(\Sigma_{i} x_{i}\right)=\bigcup_{i}\left(d x_{i}\right)$, is a partial monoid in which every family is summable.

Definition 2.7. Let $(M, \Sigma)$ and $\left(M^{\prime}, \Sigma^{\prime}\right)$ be partial monoids. Then $\left(M^{\prime}, \Sigma^{\prime}\right)$ is said to be a partial submonoid of $(M, \Sigma)$ if:

(i) $M^{\prime}$ is a subset of $M$; and

(ii) $\left(x_{i}: i \in I\right)$ is a summable family in $M^{\prime}$ implies that $\left(x_{i}: i \in I\right)$ is a summable family in $M$ and $\Sigma_{i}^{\prime} x_{i}=\Sigma_{i} x_{i}$.

If $(M, \Sigma)$ is any partial monoid, then any subset $M^{\prime}$ of $M$ which is closed under the restriction of $\Sigma$ is a partial sub-monoid of $M$.

Definition 2.8. Let $(M, \Sigma)$ and $\left(M^{\prime}, \Sigma^{\prime}\right)$ be partial monoids. Then a function $\theta: M \rightarrow M^{\prime}$ is said to be an additive map of $(M, \Sigma)$ into $\left(M^{\prime}, \Sigma^{\prime}\right)$ if $\left(x_{i}: i \in I\right)$ is a summable family in $M$ implies $\left(x_{i} \theta: i \in I\right)$ is a summable family in $M^{\prime}$ and $\left(\Sigma_{i} x_{i}\right) \theta=\Sigma_{i}^{\prime}\left(x_{i} \theta\right)$. 
Observation 2.9. If $M_{1}$ and $M_{2}$ are partial monoids and if $\theta: M_{1} \rightarrow M_{2}$ is an additive map, then $0 \theta=0$.

Products 2.10. Let $\left(\left(M^{i}, \Sigma^{i}\right): i \in I\right)$ be a family of partial monoids. Their product is the partial monoid $\left(\prod_{i \in I} M^{i}, \Sigma\right)$ together with the projection maps $\operatorname{pr}_{i}:\left(\prod_{i \in I} M^{i}, \Sigma\right) \rightarrow\left(M^{i}, \Sigma^{i}\right)$, defined as follows:

The set $\prod_{i \in I} M^{i}$ is the cartesian product of $M^{i}{ }^{\prime}$ s. Let $\left(x_{j}: j \in J\right)$ be a family in $\prod_{i \in I} M^{i}$. Then each $x_{j}=\left(x_{j}^{i}: i \in I\right)$, where $x_{j}^{i} \in M^{i}$. The family $\left(x_{j}: j \in J\right)$ is summable in $\prod_{i \in I} M^{i}$ if for each $i \in I,\left(x_{j}^{i}: j \in J\right)$ is summable in $M^{i}$, in which case $\Sigma\left(x_{j}: j \in J\right)=\Sigma\left(\left(x_{j}^{i}: i \in I\right): j \in J\right)=\left(\Sigma^{i}\left(x_{j}^{i}: j \in J\right): i \in I\right)$.

Each of the projection maps $\operatorname{pr}_{i}:\left(\prod_{i \in I} M^{i}, \Sigma\right) \rightarrow\left(M^{i}, \Sigma^{i}\right): x \rightarrow x^{i}$ is an additive map.

Definition 2.11. Let $M$ be a partial monoid, and let $E$ be an equivalence relation on the elements of $M$. Then $E$ is a partial monoid congruence on $M$ if $E$ is closed under the additive operation of the product partial monoid $M \times M$.

Definition 2.12. A partial semiring is a quadruple $(R, \Sigma, \cdot, 1)$ where $(R, \Sigma)$ is a partial monoid, $(R, \cdot, 1)$ is an ordinary monoid with multiplicative binary operation '.' and unit 1, and the additive and multiplicative structures obey the following distributive laws: If $\Sigma\left(x_{i}: i \in I\right)$ is defined in $R$, then for all $y$ in $R, \Sigma_{i} y \cdot x_{i}$ and $\Sigma_{i} x_{i} \cdot y$ are defined and $y \cdot\left(\Sigma_{i} x_{i}\right)=\Sigma_{i} y \cdot x_{i} ;\left(\Sigma_{i} x_{i}\right) \cdot y=\Sigma_{i} x_{i} \cdot y$.

Definition 2.13. A nonempty set $S$ together with a binary operation, called addition and a ternary multiplication, denoted by juxtaposition, is said to be a ternary semiring if $S$ is an additive commutative semigroup satisfying the following conditions:

(i) $(a b c) d e=a(b c d) e=a b(c d e)$,

(ii) $(a+b) c d=a c d+b c d$,

(iii) $a(b+c) d=a b d+a c d$,

(iv) $a b(c+d)=a b c+a b d$; for all $a, b, c, d, e \in S$. 


\section{Ternary Partial Semirings}

We introduce the notion of Ternary partial semiring as follows:

Definition 3.1. A nonempty set $S$ together with a partial addition $\Sigma$, defined on some (not necessarily all) families $\left(a_{i}: i \in I\right.$ ) in $S$ and a ternary multiplication, denoted by juxtaposition, is said to be a ternary partial semiring if it satisfies the following axioms:

(i) $(S, \Sigma)$ is a partial monoid,

(ii) $(a b c) d e=a(b c d) e=a b(c d e)$,

(iii) a family $\left(a_{i}: i \in I\right)$ is summable in $S$ implies $\left(a_{i} a b: i \in I\right)$ is summable in $S$ and $\left(\Sigma_{i \in I} a_{i}\right) a b=\Sigma_{i \in I}\left(a_{i} a b\right)$,

(iv) a family $\left(a_{i}: i \in I\right)$ is summable in $S$ implies $\left(a a_{i} b: i \in I\right)$ is summable in $S$ and $a\left(\Sigma_{i \in I} a_{i}\right) b=\Sigma_{i \in I}\left(a a_{i} b\right)$,

(v) a family $\left(a_{i}: i \in I\right)$ is summable in $S$ implies $\left(a b a_{i}: i \in I\right)$ is summable in $S$ and $a b\left(\Sigma_{i \in I} a_{i}\right)=\Sigma_{i \in I}\left(a b a_{i}\right)$;

(vi) $0 a b=a 0 b=a b 0=0$ where $0=\Sigma\left(a_{i}: i \in \emptyset\right)$ (empty family), acts as an additive zero for binary sums in $S$, for all $a, b, c, d, e \in S$.

Now we give some examples of ternary partial semirings.

Example 3.2. Let $S=\mathbb{Q}^{-} \bigcup\{0\}$ be the set of all nonpositive rational numbers. Then with $\Sigma$, defined as the usual addition over families of finite support and with usual ternary multiplication, $S$ forms a ternary partial semiring.

Example 3.3. Let $S^{\prime}=\mathbb{Z}^{-} \bigcup\{0\}$ be the set of all nonpositive integers. Then $S^{\prime}$ together with $\Sigma$, defined as the usual addition over families of finite support and with usual ternary multiplication, is a ternary partial semiring.

Example 3.4. The set $\mathbb{N} \bigcup\{0\}$ for all nonnegative integers is a ternary partial semiring with $\Sigma$, defined as the usual addition over families of finite support and with usual ternary multiplication.

Example 3.5. Let $\mathbb{Q}^{+}$be the set of all positive rational numbers and $S=\left\{q \sqrt{2}: q \in \mathbb{Q}^{+} \bigcup\{0\}\right\}$. Then with $\Sigma$, defined as the usual addition over families of finite support and with usual ternary multiplication, $S$ becomes a ternary partial semiring.

Example 3.6. Suppose $\mathbb{M}$ is the set of all $m \times m$ square matrices over nonpositive integers. Then $\mathbb{M}$ becomes a ternary partial semiring with normal addition over families of finite support and ternary multiplication. 
Example 3.7. Let $D$ be any set. Then $\operatorname{Pfn}(D, D)$ together with the partial summation, defined as in example 2.5 and the ternary multiplication, defined as $d(f g h)=(((d f) g) h)$, for all $d \in D$ and $f, g, h \in P f n(D, D)$, is a ternary partial semiring.

Throughout this paper, $S$ stands for a ternary partial semiring unless otherwise stated.

Definition 3.8. A partial submonoid $A$ of $S$ is called a ternary partial subsemiring if $a_{1} a_{2} a_{3} \in A$ for all $a_{1}, a_{2}, a_{3} \in A$.

Example 3.9. Consider the ternary partial semirings $S$ and $S^{\prime}$, given in example 3.2 and 3.3. Now $S^{\prime}$ is a ternary partial subsemiring of $S$.

\section{Homomorphisms and Congruence Relations of Ternary Partial Semirings}

Definition 4.1. Let $S$ and $S^{\prime}$ be two ternary partial semirings. A function $\alpha: S \rightarrow S^{\prime}$ is said to be a homomorphism if it satisfies the following conditions:

(i) If $\left(a_{i}: i \in I\right)$ is a summable family in $(S, \Sigma)$, then $\left(\alpha\left(a_{i}\right): i \in I\right)$ is a summable family in $\left(S^{\prime}, \Sigma^{\prime}\right)$, and $\alpha\left(\Sigma_{i} a_{i}\right)=\Sigma_{i}^{\prime} \alpha\left(a_{i}\right)$. (In this case, $\alpha$ is called an additive map of $(S, \Sigma)$ into $\left.\left(S^{\prime}, \Sigma^{\prime}\right)\right)$.

(ii) $\alpha(a b c)=\alpha(a) \alpha(b) \alpha(c)$ for all $a, b, c \in S$.

Note that the identity map on a ternary partial semiring is a homomorphism of ternary partial semirings. We also note that if $\alpha$ is a homomorhism of ternary partial semirings, then $\alpha(0)=0$.

Definition 4.2. Let $S$ and $S^{\prime}$ be two ternary partial semirings. A homomorphism $\alpha: S \rightarrow S^{\prime}$ is said to be a:

(i) monomorphism if $\alpha$ is one-to-one.

(ii) epimorphism if $\alpha$ is onto.

(iii) isomorphism if $\alpha$ is both one-to-one and onto.

If $S$ and $S^{\prime}$ are ternary partial semirings and if $\alpha: S \rightarrow S^{\prime}$ is a homomorphism then $\alpha(S)$ need not be closed under the partial addition of $S^{\prime}$. Hence, $\alpha(S)$ together with the partial addition of $S^{\prime}$ is not necessarily a ternary partial subsemiring of $S^{\prime}$. 
In the following example, we give two ternary partial semirings $S$ and $S^{\prime}$ and a homomorphism $\alpha: S \rightarrow S^{\prime}$ such that $\alpha(S)$ is not closed under the partial addition of $S^{\prime}$.

Example 4.3. Let $S=\{0,1\}$. For any family $\left(a_{i}: i \in I\right)$ of elements of $S$, define

$$
\Sigma_{i} x_{i}=\left\{\begin{array}{l}
0, \text { if } a_{i}=0 \text { for all } i \in I \\
1, \text { if } a_{j}=1, \text { for some } j \& a_{i}=0 \text { for all } i \in I \text { with } i \neq j \\
\text { undefined, otherwise. }
\end{array}\right.
$$

For any $a, b, c \in S$, define

$$
a b c=\left\{\begin{array}{l}
1, \text { if } a \neq 0, b \neq 0 \text { and } c \neq 0 \\
0, \text { otherwise }
\end{array}\right.
$$

Then with the partial summation and ternary multiplication, $S$ becomes a ternary partial semiring.

Consider the ternary partial semiring $S^{\prime}=\mathbb{Z}^{-} \bigcup\{0\}$, given in example 3.3.

Define $\alpha: S \rightarrow S^{\prime}$ by $\alpha(0)=0$ and $\alpha(1)=-1$. Now $\alpha$ is a partial ternary semiring homomorphism of $S$ into $S^{\prime}$. But $\alpha(1)+\alpha(1)=-1+(-1)=-2 \notin$ $\{0,-1\}=\alpha(S)$. Therefore, $\alpha(S)$ is not closed under the partial summation of $S^{\prime}$ and hence, $\alpha(S)$ not a ternary partial subsemiring.

Products 4.4. : Let $\left(S^{i}, \Sigma^{i}, f^{i}\right)$ be a family of ternary partial semirings indexed by $I$, where $f^{i}$ denotes a ternary multiplication on $S^{i}, i \in I$. Let $S$ be the Cartesian product of $S^{i}, i \in I$.

That is, $S=X_{i \in I} S^{i}$. Define a partial summation $\Sigma$, and a ternary multiplication $f$ on $S$ as follows: Let $\left(a_{j}: j \in J\right)$ be a family of elements in $S$. Then each $a_{j}$ is a tuple of of the form $a_{j}=\left(a_{j}^{i}: i \in I\right)$, where $a_{j}^{i}$ is in $S^{i}$. If, for each $i \in I,\left(a_{j}^{i}: j \in J\right)$ is summable in $S^{i}$, then the family $\left(a_{j}: j \in J\right)$ is summable in $S$, and $\Sigma\left(a_{j}^{*}: j \in J\right)=\Sigma\left(\left(a_{j}^{i}: i \in I\right): j \in J\right)=\left(\Sigma^{i}\left(a_{j}^{i}: j \in J\right): i \in I\right)$. For any three elements $a=\left(a^{i}: i \in I\right), b=\left(b^{i}: i \in I\right)$ and $c=\left(c^{i}: i \in I\right)$ in $S$, $a b c=f(a, b, c)=\left(f^{i}\left(a^{i}, b^{i}, c^{i}\right): i \in I\right)=\left(a^{i} b^{i} c^{i}: i \in I\right)$.

Then $(S, \Sigma, f)$ is a ternary partial semiring. This is called the product ternary partial semiring, and is denoted by $\prod_{i \in I} S_{i}$. Thus $S=\prod_{i \in I} S_{i}$.

Let $\left(S^{i}, \Sigma^{i}, f^{i}\right)$ be a family of ternary partial semirings indexed by $I$. Then for each $i \in I$, the mapping $\pi_{i}: \prod_{i \in I} S^{i} \rightarrow S^{i}$, defined by $\Pi_{i}(a)=a^{i}\left(i^{\text {th }}\right.$ 
component of $a$ ) is a ternary partial semiring homomorphism. This $\pi_{i}$ is called the $i^{\text {th }}$ projection map of $\left(\prod_{i \in I} S^{i}, \Sigma, f\right)$ into $\left(S^{i}, \Sigma^{i}, f^{i}\right)$ for all $i \in I$.

Definition 4.5. Let $S$ be a ternary partial semiring and let $\theta$ be a binary relation defined on $S$. Then $\theta$ is called a ternary partial semiring congruence relation on $S$. If it satisfies the following conditions:

(1) $\theta$ is an equivalence relation on $S$,

(2) $\theta$ is closed under the partial summation and the ternary operation of the product partial ternary semiring $S \times S$. That is,

(i) If $\left(a_{i}: i \in I\right)$ and $\left(b_{i}: i \in I\right)$ are summable families in $S$ such that $a_{i} \theta b_{i}$ for all $i \in I$, then $\left(\Sigma_{i} a_{i}\right) \theta\left(\Sigma_{i} b_{i}\right)$ or $\Sigma_{i}\left(a_{i}, b_{i}\right) \in \theta$,

(ii) If $a \theta a^{\prime}, b \theta b^{\prime}$ and $c \theta c^{\prime}$ where $a, b, c, a^{\prime}, b^{\prime}, c^{\prime} \in S$, then $(a b c) \theta\left(a^{\prime} b^{\prime} c^{\prime}\right)$.

Definition 4.6. Let $S$ be a ternary partial semiring. Then the congruence relation $\theta$ on $S$, defined by $s \theta s^{\prime}$ if and only if $s=s^{\prime}$ for all $s, s^{\prime} \in S$ is the trivial congruence relation and all other congruence relations are called nontrivial.

If $s \theta s^{\prime}$ for all $s, s^{\prime} \in S$, then $\theta$ is called improper and all other congruence relations are called proper.

Lemma 4.7. Let $S$ and $S^{\prime}$ be ternary partial semirings and let $\alpha: S \rightarrow S^{\prime}$ be a homomorphism. Define $a \theta b \Leftrightarrow \alpha(a)=\alpha(b)$ for all $a, b \in S$. Then $\theta$ is a ternary partial semiring congruence relation on $S$.

Proof. It is clear that $\theta$ is an equivalence relation on $S$. Let $\left(a_{i}: i \in I\right)$ and $\left(b_{i}: i \in I\right)$ be summable families in $S$ such that $\left(a_{i}, b_{i}\right) \in \theta$ for all $i \in I$. Then $\Sigma_{i} a_{i}$ and $\Sigma_{i} b_{i}$ exists in $S$ and $\alpha\left(a_{i}\right)=\alpha\left(b_{i}\right)$ for all $i \in I$ and so $\Sigma_{i}^{\prime} \alpha\left(a_{i}\right)=$ $\Sigma_{i}^{\prime} \alpha\left(b_{i}\right)$ and that $\alpha\left(\Sigma_{i} a_{i}\right)=\alpha\left(\Sigma_{i} b_{i}\right)$ and implies that $\left(\Sigma_{i} a_{i}, \Sigma_{i} b_{i}\right) \in \theta$ and hence $\Sigma_{i}\left(a_{i}, b_{i}\right) \in \theta$. Suppose that $a \theta a^{\prime}, b \theta b^{\prime}$ and $c \theta c^{\prime}$ where $a, b, c, a^{\prime}, b^{\prime}, c^{\prime} \in S$. Then $\alpha(a)=\alpha\left(a^{\prime}\right), \alpha(b)=\alpha\left(b^{\prime}\right)$ and $\alpha(c)=\alpha\left(c^{\prime}\right)$. So, $\alpha(a b c)=\alpha(a) \alpha(b) \alpha(c)=$ $\alpha\left(a^{\prime}\right) \alpha\left(b^{\prime}\right) \alpha\left(c^{\prime}\right)=\alpha\left(a^{\prime} b^{\prime} c^{\prime}\right)$. This implies that $(a b c) \theta\left(a^{\prime} b^{\prime} c^{\prime}\right)$. Hence, $\theta$ is a ternary partial semiring congruence relation on $S$.

Construction 4.8. Let $S$ be a ternary partial semiring and let $\theta$ be a ternary partial semiring congruence relation on $S$.

For $s \in S$, let $\theta s$ denote the equivalence class containing $s$ with respect to $\theta$. We write $S / \theta=\{\theta s \mid s \in S\}$. The natural addition $\widehat{\Sigma}$ and natural ternary multiplicative operation on $S / \theta$ are defined as follows: For any family 
$\left(\theta a_{i}: i \in I\right)$ of elements in $S / \theta$, define

$$
\widehat{\Sigma}_{i} \theta a_{i}=\left\{\begin{array}{l}
\theta\left(\Sigma_{i} a_{i}\right), \text { if } \Sigma_{i} a_{i}, \text { exists in } S \\
\text { undefined, otherwise. }
\end{array}\right.
$$

For any $\theta a, \theta b, \theta c \in S / \theta$, define $\theta a \theta b \theta c=\theta(a b c)$. However, there is no guarantee that $S / \theta$ is a ternary partial semiring with respect to the above natural addition and the natural ternary multiplicative operation.

In the following example, we show that if $S$ is a ternary partial semiring and $\theta$ is a congruence relation on $S$, then $S / \theta$ is not a ternary partial semiring with respect to the natural addition and the natural ternary multiplication.

Example 4.9. Let $\mathfrak{P}(D)$ be the power set of a set $D$. For any family $\left(A_{i}: i \in I\right)$ of elements in $\mathfrak{P}(D)$, define

$$
\Sigma_{i} A_{i}=\left\{\begin{array}{l}
\bigcup A_{i} \text { (usual set union), if } A_{i} \bigcap A_{j}=\phi \forall i \neq j \\
\text { undefined, otherwise }
\end{array}\right.
$$

For any three elements $A, B, C \in \mathfrak{P}(D)$, define $A B C=A \bigcap B \bigcap C$ (Usual set intersection). Then $\mathfrak{P}(D)$ together with the above partial summation and the ternary multiplication, forms a ternary partial semiring. Take $D=\{x, y\}$ and $\theta=\{(\emptyset, \emptyset),(\{x\},\{x\}),(\{y\},\{y\}),(D, D),(\{x\}, D),(D,\{x\}),(\emptyset,\{y\}),(\{y\}, \emptyset)\}$.

Then $\theta$ is a ternary partial semiring congruence relation on $\mathfrak{P}(D)$. Now $\mathfrak{P}(D) / \theta=\{\bar{\emptyset}, \overline{\{x\}}\}$ where $\bar{\emptyset}=\{\emptyset,\{y\}\}=\overline{\{y\}}, \overline{\{x\}}=\{\{x\}, D\}=\bar{D}$. In this case, $\{x\}+\{y\}=D$ is defined in $\mathfrak{P}(D)$ but $\overline{\{x\}}+\overline{\{y\}}$ is not well defined in $\mathfrak{P}(D) / \theta$, as $D+\{y\}$ is not defined in $\mathfrak{P}(D)$. Hence, $\mathfrak{P}(D) / \theta$ is not a ternary partial semiring.

The following is a necessary and sufficient condition for $S / \theta$ to be a ternary partial semiring where $S$ is a ternary partial semiring and $\theta$ is a ternary partial semiring congruence relation on $S$.

If $\left(a_{i}: i \in I\right)$ is a summable family in $S$ and $\left(b_{i}: i \in I\right)$ is any family in $S$ with $a_{i} \theta b_{i} \forall i \in I$, then $\left(b_{i}: i \in I\right)$ is summable in $S$.

Theorem 4.10. Let $S$ be a ternary partial semiring and $\theta$ be a ternary partial semiring congruence relation on $S$. Suppose that $\left(a_{i}: i \in I\right)$ and $\left(b_{i}: i \in I\right)$ are any two families in $S, a_{i} \theta b_{i} \forall i \in I$ and $\left(a_{i}: i \in I\right)$ is summable in $S$ implies $\left(b_{i}: i \in I\right)$ is summable in $S$. Then $S / \theta$ is a ternary partial semiring.

Proof. First we show that if $\left(a_{i}: i \in I\right)$ is a summable family in $S$, then $\left(\theta a_{i}: i \in I\right)$ is a summable family in $S / \theta$. Let $\left(a_{i}: i \in I\right)$ be a summable family 
in $S$. For $i \in I$, let $b_{i} \in \theta a_{i}$. Now $\left(b_{i}: i \in I\right)$ is a family of elements of $S$ such that $a_{i} \theta b_{i} \forall i \in I$. Since $\left(a_{i}: i \in I\right)$ is summable in $S$ and $a_{i} \theta b_{i} \forall i \in I$, by hypothesis, $\left(b_{i}: i \in I\right)$ is summable in $S$. Therefore $\left(\theta a_{i}: i \in I\right)$ is summable in $S / \theta$, since $\left(b_{i}: i \in I\right)$ was arbitrary.

Now we show that $S / \theta$ is a ternary partial semiring with the natural partial summation $\widehat{\Sigma}$ and the natural ternary multiplication.

(i) Unary sum axiom: If $\left(\theta a_{i}: i \in I\right)$ is a one element family in $S / \theta$ and $I=\{j\}$, then $\left(a_{i}: i \in I\right)$ is a one element family in $S$ and $I=\{j\}$ and hence $\Sigma\left(a_{i}: i \in I\right)$ exists in $S$ and equals $a_{j}$ since $S$ is a partial monoid and implies that $\widehat{\Sigma}\left(\theta a_{i}: i \in I\right)$ exists in $S / \theta$ and equals $\theta a_{j}$.

(ii) Partition associativity axiom: Let $\left(\theta a_{i}: i \in I\right)$ be a summable family in $S / \theta$ and $\left(I_{j}: j \in J\right)$ be a partition of $I$. Then $\left(a_{i}: i \in I\right)$ is a summable family in $S$ and $\left(I_{j}: j \in J\right)$ is a partition of $I$ and hence each family $\left(a_{i}: i \in I_{j}\right)$ is summable in $S$ for $j \in J$ and the family $\left(\left(\Sigma a_{i}: i \in I_{j}\right): j \in J\right)$ is summable in $S$, and also $\left(\Sigma a_{i}: i \in I\right)=\Sigma\left(\Sigma\left(a_{i}: i \in I_{j}\right): j \in J\right)$.

This implies that each family $\left(\theta a_{i}: i \in I_{j}\right)$ is summable in $S / \theta$ for $j \in J$ and the family $\left(\widehat{\Sigma}\left(\theta a_{i}: i \in I_{j}\right): j \in J\right)$ is summable in $S / \theta$, and also $\widehat{\Sigma}\left(\theta a_{i}\right.$ : $i \in I)=\widehat{\Sigma}\left(\widehat{\Sigma}\left(\theta a_{i}: i \in I_{j}\right): j \in J\right)$.

Therefore, $S / \theta$ is a partial monoid. For any $\theta a, \theta b, \theta c$ in $S / \theta$, we have $\theta a \theta b \theta c=\theta(a b c)$. For any $\theta a, \theta b, \theta c, \theta d, \theta e$ in $S / \theta(\theta a \theta b \theta c) \theta d \theta e=\theta(a b c) \theta d \theta e=$ $\theta((a b c) d e)=\theta(a(b c d) e)=\theta a \theta(b c d) \theta e=\theta a(\theta b \theta c \theta d) \theta e$, and $(\theta a \theta b \theta c) \theta d \theta e=$ $\theta a \theta b \theta(c d e)=\theta a \theta b(\theta c \theta d \theta e)$. Let $\left(\theta a_{i}: i \in I\right)$ be a summable family in $S / \theta$ and $\theta a, \theta b \in S / \theta$. Then $\left(a_{i}: i \in I\right)$ is a summable family in $S$ and $a, b \in S$, and hence $\left(a_{i} a b: i \in I\right)$ is a summable family in $S$ and $\left(\Sigma\left(a_{i}: i \in I\right)\right) a b=$ $\Sigma\left(a_{i} a b: i \in I\right)$. This implies that $\left(\theta\left(a_{i} a b\right): i \in I\right)$ is a summable family in $S / \theta$ and $\theta\left(\left(\Sigma\left(a_{i}: i \in I\right)\right) a b\right)=\theta\left(\Sigma\left(a_{i} a b: i \in I\right)\right)$ and that $\left(\theta\left(a_{i} a b\right): i \in I\right)$ is a summable family in $S / \theta$ and $\left(\theta\left(\Sigma\left(a_{i}: i \in I\right)\right) \theta a \theta b\right)=\widehat{\Sigma}\left(\theta\left(a_{i} a b\right): i \in I\right)$ and that $\left(\theta a_{i} \theta a \theta b: i \in I\right)$ is a summable family in $S / \theta$ and $\left(\widehat{\Sigma} \theta a_{i}: i \in I\right) \theta a \theta b=$ $\widehat{\Sigma}\left(\theta a_{i} \theta a \theta b: i \in I\right)$. Similarly, $\left(\theta a \theta a_{i} \theta b: i \in I\right)$ is a summable family in $S / \theta$ and $\theta a\left(\widehat{\Sigma}\left(\theta a_{i}: i \in I\right)\right) \theta b=\widehat{\Sigma}\left(\theta a \theta a_{i} \theta b: i \in I\right)$ and $\left(\theta a \theta b \theta a_{i}: i \in I\right)$ is a summable family in $S / \theta$ and $\theta a \theta b\left(\widehat{\Sigma}\left(\theta a_{i}: i \in I\right)\right)=\widehat{\Sigma}\left(\theta a \theta b \theta a_{i}: i \in I\right)$. Also, $\theta 0 \theta a \theta b=\theta(0 a b)=\theta 0, \theta a \theta 0 \theta b=\theta(a 0 b)=\theta 0$ and $\theta a \theta b \theta 0=\theta(a b 0)=\theta 0$. Therefore $\theta 0 \theta a \theta b=\theta a \theta 0 \theta b=\theta a \theta b \theta 0=0$ in $S / \theta$. Hence, $S / \theta$ is a ternary partial semiring.

\section{References}

[1] Acharyulu, G.V.S., Matrix Representable So-rings, Semigroup Forum, Vol. 46, no.1, pp. 37-47, Springer-Verlag, 1993. http://dx.doi.org/10.1007/BF02573542 
[2] Acharyulu, G.V.S., A Study of Sum-Ordered Partial Semirings, Doctoral thesis, Andhra University, 1992.

[3] Arbib, M.A., Manes, E.G., Partially Additive Categories and Flow-diagram Semantics, Journal of Algebra, Vol. 62, no.1, pp. 203-227, 1980. http://dx.doi.org/10.1016/00218693(80)90212-4

[4] Allan, P. J., A fundamental theorem of homomorphisms for semirings, Proc. Amer. Math. Soc., Vol. 21, no.2, pp. 412-416, 1969. http://dx.doi.org/10.1090/S0002-9939-1969$0237575-4$

[5] Dutta, T.K., Kar, S., On regular ternary semirings, Advances in Algebra, Proceedings of the ICM, Satellite conference in Algebra and Related Topics, World Scientific, pp. 343-355, 2003. http://dx.doi.org/10.1142/9789812705808-0027

[6] Dutta, T.K., Kar, S., On the Jacobson radical of a ternary semiring, Southeast Asian Bulletin of Mathematics, Vol. 28, No. 1, pp. 1-13, 2004.

[7] Golan, Janathan, S., Semirings and their applications, Kluwer Academic Publishers, Dordrecht (1999). http://dx.doi.org/10.1007/978-94-015-9333-5

[8] Lehmer, D. H., A ternary analogue of abelian groups, American Journal of Mathematics, Vol. 59, no.2, pp. 329, 1932. http://dx.doi.org/10.2307/2370997

[9] Lister, W. G., Ternary Rings, Trans. Amer. Math. Soc., 154(1971), 3755. http://dx.doi.org/10.1090/S0002-9947-1971-0272835-6

[10] Manes, E. G., Arbib, M. A., Algebraic Approaches to program semantics, Springer-verlag, Newyork, 1985. http://dx.doi.org/10.1007/978-1-4612-4962-7

[11] Manes, E.G., and Benson, D.B., The Inverse Semigroup of a Sum-Ordered Partial Semiring, Semigroup Forum, Vol. 31, pp. 129-152, 1985.

[12] Streenstrup, M.E., Sum-Ordered Partial Semirings, Doctoral thesis, Graduate school of the University of Massachusetts, 1985.

[13] Walendziak, A., A Common Generalization of Subdirect and Direct product of Algebras, Annales Societatis Mathematicae Polanae, Series I, Commentations Mathemathicae, Vol. 17, 1992. 
